\title{
Pressurised solvent extraction of environmental organic compounds in soils using a supercritical fluid extractor
}

\author{
K. Li ${ }^{1}$, M. Landriault ${ }^{1}$, M. Fingas ${ }^{1}$ and M. Llompart ${ }^{2, *}$ \\ ${ }^{1}$ Emergencies Science Division, Environment Canada, Environmental Technology Centre, \\ River Road, Ottawa, Ontario, Canada \\ ${ }^{2}$ Departamento de Quimica Analitica, Nutricion y Bromatologia, Facultad de Quimica, \\ Universidad de Santiago de Compostela, E-15706 Santiago de Compostela, Spain
}

\begin{abstract}
The applicability of pressurised solvent extraction (PSE) for the quantitative extraction of different classes of semivolatiles, including polycyclic aromatic hydrocarbons (PAHs), phenols, polychlorinated biphenyls (PCBs) and total petroleum hydrocarbons have been evaluated. For this study a conventional supercritical fluid extraction (SFE) system, the Suprex SFE/50 was adapted to function as a pressurised solvent extraction system. Solid samples were weighed into the SFE thimble and extracted using conventional extraction solvents instead of supercritical carbon dioxide. Parameters such as extraction temperature and effect of modifiers were investigated. Although limited by the $150^{\circ} \mathrm{C}$ maximum oven temperature, it was found effective extraction could still be carried out in less than 25 min for all the compounds studied. The technique was applied to different real matrices contaminated with hydrocarbons, PAHs and phenols. Validations of the technique were performed using standard reference materials. Recoveries for these matrices were good (> 75\%) and precision was generally less than a 10\% RSD. Extensive comparison of this technique with sonication and with microwave assisted extraction (MAE) were made, and recoveries were found to be comparable to MAE and superior to sonication.
\end{abstract}

Key words. Pressurised solvent extraction - microwave assisted extraction - PAHs - PCBs - phenols - total petroleum hydrocarbons - soil samples.

\section{Introduction}

The desire to reduce the use of hazardous organic solvents in analytical extraction has contributed in the last few years to the development of new technologies using less solvent than classical extraction procedures. Among the new techniques, supercritical fluid extraction (SFE) and microwave assisted extraction (MAE) have achieved great popularity, evident from the number of applications in environmental analysis [1-9]. SFE reduces the usage of hazardous solvents because it uses carbon dioxide as the principal extraction fluid [1-6]. MAE can also reduce solvent usage and shorten extraction times [6-9].

A recent advance in sample preparation for trace environmental analysis is the pressurised solvent extraction (PSE). This technique uses conventional solvents at elevated pressures and temperatures to extract solid samples quickly [10-14]. The process takes advantage of the increased analyte solubilities at temperatures well above the boiling points of common solvents. At higher temperatures the kinetic processes for the desorption of analytes from the matrix are accelerated. Currently a commercial unit is available in which automated extractions can be carried out on 24 samples sequentially $[10,12]$. This technique offers some advantages over SFE and MAE. SFE uses supercritical $\mathrm{CO}_{2}$, which is a nonpolar fluid. For the extraction of polar compounds, even after the addition of a polar modifier to the $\mathrm{CO}_{2}$, very high temperatures and pressures are sometimes necessary to achieve a quantitative extraction of polar analytes [15], which increases instrument wear and analytical cost. In MAE, extraction of solid samples is effected by rapid heating of the polar solvent by microwave energy. Instrumentation is relatively simple and method development time is generally reduced. It is, however, subject to interferences from the presence of microwave energy absorbing materials in the sample such as ferrous materials and charcoal which can cause arcing. At the end of the extraction, extraction vessels must be cooled to room temperature before they can be opened, which increases the overall extraction time. In addition, MAE requires filtration to separate the raw extract from the solid material once the extraction finished.

In this work we studied the feasibility of performing PSE using an SFE instrument as well as the ability of PSE to extract different groups of semivolatiles (PAHs, PCBs, hydrocarbons and phenols) from soil samples. For this study, a supercritical fluid extraction (SFE) system, the Suprex SFE/50 was adapted to operate as a PSE system. Different parameters affecting the extraction efficiency were investigated. Extensive comparison of this technique with sonication and with MAE was made. Four real contaminated samples were extracted to establish the validity of the proposed method.

\section{Experimental}

\section{Reagents and materials}

Analytical reference standard solution (SRM 1491) of the target PAHs was obtained from NIST (Gaithersburg, MD). Working solutions were prepared in hexane. A surrogate 
mixture of four deuterated PAH compounds was purchased from Supelco (Mississauga, Ontario, Canada).

For hydrocarbon fuel analysis, standards were made by diluting one $\mu \mathrm{L}$ of the fuel in $1 \mathrm{~mL}$ of hexane/acetone.

For PCB analysis, analytical reference standard solution of Aroclor 1260 was obtained from Ultrascience (Den Mills, Ontario, Canada).

Phenol standards were supplied by Aldrich Chemie (Steinheim, Germany). Standard stock solutions were prepared by weighing an appropriate amount of each standard and diluting to $10 \mathrm{~mL}$ with hexane. Working solutions were made by appropriate dilution of the stock solutions.

All solvents were of distilled-in-glass grade and purchased from Caledon (Belleville, Canada).

Four real contaminated matrices were used in this study. The SRS103-100 certified reference material is a soil contaminated with PAHs and was purchased from Fisher Scientific (Fair Lawn, NJ). The SRM 1649 certified reference material is an urban dust certified for 5 PAHs and with provisional PAH values for another 4 PAHs. This material was purchased from NIST (Gaithersburg, MD). The other two materials were a diesel contaminated soil from a sour gas process plant and a natural soil from a coke plant. The cokery soil was obtained as a result of an inter-laboratory comparison exercise sponsored by the Community Bureau of Reference (BCR) of the Commission of the European Community aiming at the certification of a reference soil material for phenol and cresols. This material is heavily contaminated with cyanides, PAHs and phenols among others. More than $80 \%$ of the total phenol constituted of phenol, ocresol, m-cresol and p-cresol. This soil is considered a difficult matrix to analyse because of the high level of contamination and a strongly adsorptive matrix due to a carbon content of almost $20 \%$.

\section{Instrumentation}

The system used for PSE work was the Suprex SFE/50 system, which was a research-grade modular SFE system consisting of a syringe pump with a capacity of $240 \mathrm{~mL}$, a system controller and an extraction oven with a heated valve compartment with a 4-way valve allowing the SF carbon dioxide to be collected in different collection vessels. For this work, the system was modified with the installation of a manual static/dynamic valve down stream of the thimble, bypassing the 4-port selector valve. A cylinder of nitrogen was connected to the 3-way purge valve upstream of the thimble normally used to vent pressure of the thimble. This functioned to flush out the solvent from the thimble after each extraction. A $0.25 \mathrm{~mm}$ id stainless steel capillary was used to direct the solvent from the static valve to an open collection vessel. The syringe pump was simply filled by dipping the stainless steel line normally connected to a SFE grade carbon dioxide into a solvent bottle of hexane and using the "refill" function on the control module. Throughout this study, the pump seals made of Teflon/graphite composite material did not suffer any deterioration from exposure to the solvent.

MAE experiments were performed in a 950-W MES-1000 microwave sample preparation system using Teflon-lined extraction vessels (CEM Corp., Mathews, NC).
Ultrasonic extractions were carried out in an ultrasonic bath (Branson Ultrasonics Corp., Danbury, CT). Vials of $22 \mathrm{~mL}$ equipped with Teflon-lined septa and screw caps were used.

\section{PSE procedure}

A $0.1-2 \mathrm{~g}$ aliquot of soil was weighed into a $5-\mathrm{mL}$ stainless steel thimble; for PAH analysis an aliquot of surrogate mixture was added just prior to extraction. For most of the extractions, a polar cosolvent (acetone or methanol) was added just before extraction. The end caps of the cell were screwed on and hand tightened. The syringe pump then delivered the solvent to the thimble and pressurised the system to $100 \mathrm{~atm}$. Simultaneously, the oven was heated up to $50-150{ }^{\circ} \mathrm{C}$. The solvent used was hexane. After reaching the experimental conditions $(5-10 \mathrm{~min})$, the sample was kept in this pressurised fluid environment for $15 \mathrm{~min}$. Then, the pump was stopped and the system was depressurised by opening the static valve, forcing the raw extract out into a calibrated test tube. When the flow had ceased, the purge valve was opened to the high pressure nitrogen at $100 \mathrm{psi}$ to flush out the residual solvent from the thimble for about one minute. The system was returned briefly to a pressurised state for the pump to deliver a few $\mathrm{mL}$ of fresh solvent to the thimble to rinse the sample into the same test tube. This procedure was repeated $2-3$. The final volume of the extract collected was $10-15 \mathrm{~mL}$. The extract was analysed directly or after concentration by nitrogen blow-down.

\section{MAE procedure}

A $0.1-2 \mathrm{~g}$ aliquot of soil was weighed into the Teflon extraction vessel and $10 \mathrm{~mL}$ of hexane-acetone (1:1) were added. For PAH analysis an aliquot of surrogate mixture was added to the sample just prior the addition of the solvent. The extraction vessel was closed, after ensuring that a new rupture membrane was used for each extraction. Multiple extraction (3 - 6 samples simultaneously) was performed at $115^{\circ} \mathrm{C}$ for $20 \mathrm{~min}$ at $100 \%$ power. After extraction, the vessels were allowed to cool to room temperature before opened. The supernatant was filtered through a $45-\mu \mathrm{m}$ nylon disk. The extract was analysed. In some cases a concentration step before analysis was necessary.

\section{Sonication}

A $0.1-2 \mathrm{~g}$ aliquot of soil was weighed in a $22-\mathrm{mL}$ glass vial and all samples were extracted with $5 \mathrm{~mL}$ aliquots of hexane-acetone $(1: 1)$ for 20 minutes and the process was repeated two more times with fresh solvent $(3 \times 5 \mathrm{~mL}$ total $)$. For PAH analysis an aliquot of surrogate mixture was added to the sample just prior the addition of the solvent. The combined extract was filtered and analysed without any cleanup. In some cases a concentration step before analysis was necessary.

\section{Analysis}

PAH, PCB and phenol analysis were carried out on a Hewlett-Packard HP5890 Series II-HP/5971 MSD operated through a HP Chemstation software (G1034). A 30-m HP-1 capillary column $(0.2-\mathrm{mm}$ id, $0.3-\mu \mathrm{m}$ film) was used. Experimental parameters were: injector temperature $270{ }^{\circ} \mathrm{C}$; capillary interface temperature $300{ }^{\circ} \mathrm{C}$; automated injection 
of $1 \mu \mathrm{L}$; MSD operated in Selected Ion Monitoring (SIM) mode. Tuning was performed by autotuning and the electron multiplier was at a nominal value of $1400 \mathrm{~V}$. The oven temperature program for $\mathrm{PAH}$ analysis was: $40{ }^{\circ} \mathrm{C}$ for $1 \mathrm{~min}$ heated to $170{ }^{\circ} \mathrm{C}$ at $30{ }^{\circ} \mathrm{C} / \mathrm{min}$; heated to $240{ }^{\circ} \mathrm{C}$ at $4{ }^{\circ} \mathrm{C} / \mathrm{min}$; and finally heated to a final temperature of $300{ }^{\circ} \mathrm{C}$ at $12{ }^{\circ} \mathrm{C} / \mathrm{min}$, and held at this temperature for $10 \mathrm{~min}$. The oven temperature program for $\mathrm{PCB}$ analysis was: $120{ }^{\circ} \mathrm{C}$ for $1 \mathrm{~min}$ heated to a final temperature of $310^{\circ} \mathrm{C}$ at $10^{\circ} \mathrm{C} / \mathrm{min}$, and held at this temperature for $5 \mathrm{~min}$. The oven temperature program for phenol analysis was: $40{ }^{\circ} \mathrm{C}$ for $1 \mathrm{~min}$ heated to $130{ }^{\circ} \mathrm{C}$ at $30^{\circ} \mathrm{C} / \mathrm{min}$ and held for $4 \mathrm{~min}$. Prior to injection, an internal standard of d14-Terphenyl was added to give a final concentration of $1 \mu \mathrm{g} / \mathrm{mL}$. Quantitation was based on the internal standard method.

For total hydrocarbon analysis a $5890 \mathrm{GC}$ equipped with a flame ionisation detector (FID) was used. A DB-5 $30 \mathrm{M}$ $\times 0.25 \mathrm{~mm}$ i.d. column with $0.25-\mu \mathrm{m}$ film was used. Oven temperature was $50{ }^{\circ} \mathrm{C}$ for $1 \mathrm{~min}$ and heated to $310^{\circ}$ at the rate of $15^{\circ} \mathrm{C} / \mathrm{min}$. One $\mu \mathrm{L}$ was injected splitless via an autosampler. Injector and detector temperature was $280{ }^{\circ} \mathrm{C}$ and $310{ }^{\circ} \mathrm{C}$ respectively. The GC column flow was nominally $1 \mathrm{~mL}$ per min. Quantitation was carried out using manual integration of the unresolved hydrocarbon envelop between carbon number 12 and 22. The area total was used to compute the amount of fuel present in the sample by comparing to an external standard.

\section{Results and discussion}

\section{Preliminary experiments}

Preliminary work was performed using artificially spiked samples. The temperature and pressure were $100{ }^{\circ} \mathrm{C}$ and $100 \mathrm{~atm}$. The solvent used was hexane. The soil matrix consisted of a garden soil from an urban area, prepared by airdrying, crushing and screening to $200 \mu \mathrm{m}$ particle size. Hydrocarbon fuel mixtures (diesel and jet) as well as Aroclor 1260 were used to spike this soil. Soil concentration was at $2 \mu \mathrm{g} / \mathrm{g}$ level. Collection was made without any rinsings of the thimble. This was found to give highly variable recovery figures because of the residual solvent left in the thimble after depressurisation. The optimised collection procedure required the thimble to be rinsed $2-3$ times with several $\mathrm{mL}$ fresh solvent and purged each time using high pressure nitrogen. With this collection procedure, quantitative recoveries of the analytes spiked into the matrices were obtained. The mean of the triplicate fuel spike recovery was $82.4 \%$ and for Aroclor recovery was $98 \%$. Amount of spike found in a second extraction of the same sample was generally less than $10 \%$ of the first extraction.

\section{Effect of modifier addition}

In all our studies the extraction solvent used was hexane. The effect of adding acetone as a polar modifier was investigated. In these experiments the temperature and pressure were $100{ }^{\circ} \mathrm{C}$ and $100 \mathrm{~atm}$. The modifier can be added to the extraction cell or to the solvent container from which the syringe pump was refilled. The role of a polar modifier in PSE is similar to that in SFE, which is to increase the solubility of polar analytes, to help in weakening of the analyte-matrix interactions and to compete with the analytes for the active sites on the matrix surface. For these studies the cokery soil contaminated with PAHs and phenols was used. The level of contamination of this material is not known. The results of PAH extraction are illustrated in figure 1. For the 5-ring compounds the recoveries without the addition of a modifier were around $70 \%$ of the ones using modifier. For the 6-ring compounds the benefit of using cosolvent was more pronounced: without modifier recoveries were only $50 \%$ of those using modifier.

Due to the polar nature of phenolic compounds the addition of a polar cosolvent was essential for quantitative extraction. As can be seen in figure 2, the recoveries obtained without the addition of modifier were only 15 $30 \%$ of the recoveries using modifier. For these compounds we also study the effect using methanol instead of acetone as well as different amounts of acetone $(0.2$ and $2.5 \mathrm{~mL})$. As can be seen in figure 2 the recoveries were identical in all cases.

\section{Effect of the temperature}

The temperature study was confined to the $150{ }^{\circ} \mathrm{C}$ maximum operating temperature of the oven. For all the matrices studied in this work we found that this temperature was high enough to effect quantitative extractions of all target analytes.

The effect of the temperature was studied using standard reference material (Urban Dust SRM 1649) contaminated with PAHs. The material was extracted at temperatures of 50, 100 and $150{ }^{\circ} \mathrm{C}$ and the results are shown in table I. For this matrix, even at $50^{\circ} \mathrm{C}$ PSE produced good recoveries for most of the compounds, with the exception of the 6-ring

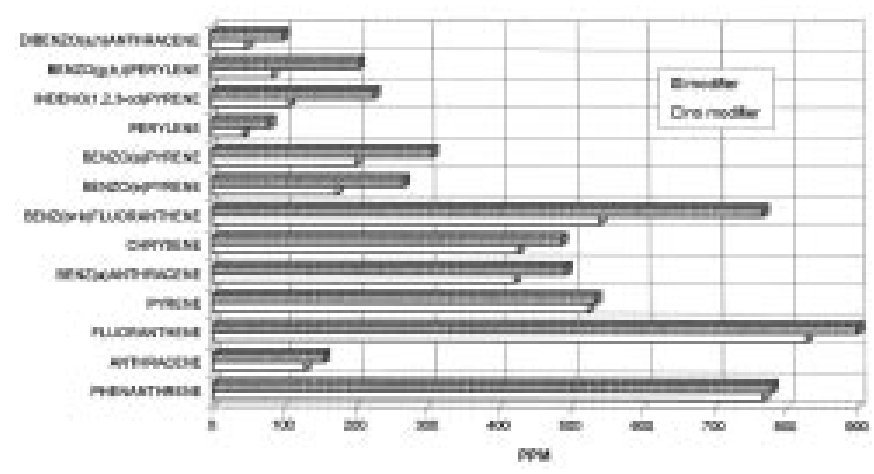

Fig. 1. PSE PAH recoveries ( $\mu \mathrm{g} / \mathrm{g}$ extracted) from cokery soil without modifier and with modifier.

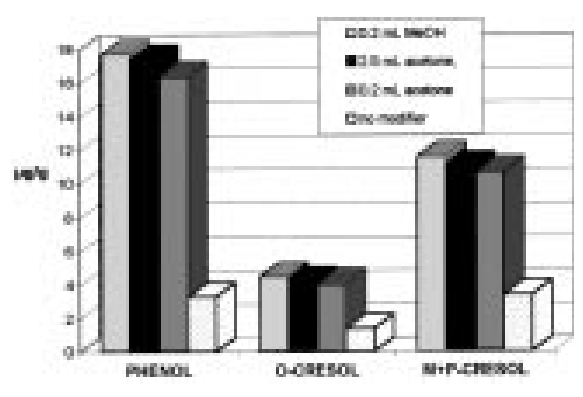

Fig. 2. PSE phenol recoveries ( $\mu \mathrm{g} / \mathrm{g}$ extracted) from cokery soil without modifier and with two different modifiers and quantities. 


\section{Original articles}

Table I. PSE percent recoveries of PAHs from urban dust SRM 1649 using different extraction temperatures. The reference values for this soil are given in table IV.

\begin{tabular}{lrrr}
\hline & \multicolumn{3}{c}{ RECOVERY (\%) } \\
& $50{ }^{\circ} \mathrm{C}$ & $100{ }^{\circ} \mathrm{C}$ & $150{ }^{\circ} \mathrm{C}$ \\
\hline PHENANTHRENE & 78.6 & 90.7 & 95.9 \\
FLUORANTHENE & 92.6 & 89.7 & 92.9 \\
PYRENE & 85.2 & 77.5 & 84.0 \\
BENZO(a)ANTHRACENE & 81.0 & 81.5 & 88.0 \\
CHRYSENE & 112.3 & 118.0 & 126.0 \\
BENZO(b,k)FLUORANTHENE & 100.7 & 105.2 & 107.5 \\
BENZO(a)PYRENE & 77.4 & 80.6 & 73.9 \\
BENZO(g,h,i)PERYLENE & 66.9 & 76.6 & 75.4 \\
INDENO(1,2,3-cd)PYRENE & 65.6 & 79.2 & 74.5 \\
\hline
\end{tabular}

Table II. PSE phenol recoveries $(\mu \mathrm{g} / \mathrm{g}$ extracted) from cokery soil using two extraction temperatures.

\begin{tabular}{lcc}
\hline & \multicolumn{2}{c}{ RECOVERY $(\mu g / g)$} \\
& $100{ }^{\circ} \mathrm{C}$ & $150{ }^{\circ} \mathrm{C}$ \\
\hline PHENOL & 12.3 & 17.4 \\
O-CRESOL & 3.4 & 4.2 \\
M+P-CRESOL & 9.2 & 11 \\
\hline
\end{tabular}

PAHs. Higher temperatures improve solubility and speed up diffusion and mass transfer and should give better extraction efficiencies for the high molecular weight PAHs. At $100{ }^{\circ} \mathrm{C}$ the recoveries for benzo(g,h,i)perylene and indeno(1,2,3cd)pyrene were $15-20 \%$ superior. The optimal working temperature was found to be $100{ }^{\circ} \mathrm{C}$, as higher temperatures did not offer better results.

We also studied the influence of the temperature in the extraction of phenols from a real contaminated cokery soil with $18 \%$ carbon content. Extractions were carried out at $100{ }^{\circ} \mathrm{C}$ and $150{ }^{\circ} \mathrm{C}$ while keeping the rest of the extraction parameters the same. As we can see in table II, the amount of phenols found at $100{ }^{\circ} \mathrm{C}$ were only $70-80 \%$ of that obtained at $150{ }^{\circ} \mathrm{C}$.

As expected, the optimum extraction temperature depends on the type of compounds as well as on the matrix but, as we have demonstrated, extraction temperatures between $100{ }^{\circ} \mathrm{C}$ and $150{ }^{\circ} \mathrm{C}$ are high enough to give good recoveries in most cases.

\section{Comparison with sonication and MAE. Validation of the method}

Extensive studies were performed to compare PSE with ultrasonic extraction as well as the relatively novel technique MAE. These studies were conducted with actual contaminated soils with three different classes of compounds. The first one was a diesel contaminated soil from a gas process plant, the other was a cokery soil heavily contaminated with PAH and phenol. For these materials no certified or reference values about the concentration of the analytes studied are available.

The results obtained for the extraction of diesel hydrocarbon are shown in table III. PSE and MAE gave similar
Table III. Total hydrocarbon recoveries ( $\mu \mathrm{g} / \mathrm{g}$ found) from diesel contaminated soil obtained using sonication, MAE and PSE. The number of determinations for each technique was 3 .

\begin{tabular}{lccc}
\hline & Sonication & MAE & PSE \\
\hline Recovery $(\boldsymbol{\mu g} / \mathbf{g})$ & 2166 & 2582 & 2727 \\
RSD $(\%)$ & 8.3 & 5.3 & 8.9 \\
\hline
\end{tabular}

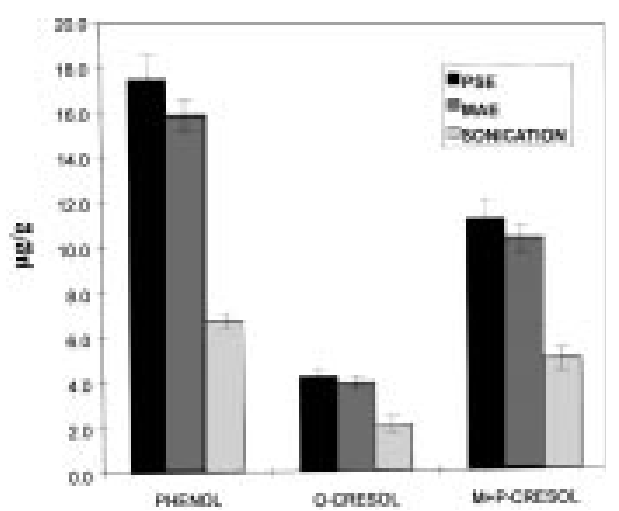

Fig. 3. Phenol recoveries ( $\mu \mathrm{g} / \mathrm{g}$ extracted) from cokery soil obtained using sonication, MAE and PSE.

recoveries. The recoveries obtained with sonication were slightly lower at about $80 \%$ of the ones obtained with the other two techniques, although this difference was not statistically significant.

The results obtained for the extraction of phenols in cokery soil are shown in figure 3. Again PSE and MAE offered same recoveries. Sonication was found to be inadequate for the extraction of phenol and cresols in this matrix, and the recoveries obtained were only $50 \%$ of the ones with PSE and MAE.

The three techniques showed similar precision, with RSD on the average around $8 \%$. The PSE method was also validated with two different certified materials: the SRM 1649 urban dust and SRS 103-100 certified reference material. The PAH results are shown in tables IV and V. PSE produced recoveries over $75 \%$. Some high recoveries were obtained for some compounds, for which reference value are not available. Figure 4 shows the total ion chromatogram (TIC) of the urban dust SRM from PSE, the prominent peaks being that of the deuterated surrogate PAHs and an internal standard. For comparison, the PAH calibration standard of $0.7 \mu \mathrm{g} / \mathrm{mL}$ is also shown.

\section{Conclusions}

Although we have not covered all classes of environmentally-important compounds, PSE of representative semivolatile compounds in different artificial matrices as well as real samples indicate this technique is a viable alternative in the new regime of rapid and low solvent consuming extraction methods such as MAE or SFE. Recoveries using PSE extraction were in all cases satisfactory, offering good agreement with the certified values and also comparable with the 
Table IV. PSE percent recoveries and precision of PAHs from SRS 103-100 certified reference material. The number of determinations was 4.

\begin{tabular}{|c|c|c|c|c|}
\hline & Reference value $(\mu \mathrm{g} / \mathrm{g})$ & Confidence interval & Recovery $(\%)$ & $R S D(\%)$ \\
\hline ANTHRACENE & 431 & $389.1-473.2$ & 91.1 & 9.3 \\
\hline PHENANTHRENE & 1925 & $1716.2-2133.6$ & 84.2 & 12.0 \\
\hline FLUORANTHENE* & 1426 & & 91.2 & 10.9 \\
\hline PYRENE & 1075 & $933.9-1216.3$ & 107.5 & 10.9 \\
\hline BENZO(a)ANTHRACENE & 264 & $240.8-288$ & 92.7 & 6.8 \\
\hline CHRYSENE & 316 & $286.5-345.8$ & 103.6 & 10.0 \\
\hline BENZO(b.k)FLUORANTHENE* & 178.4 & & 116.3 & 11.7 \\
\hline BENZO(a)PYRENE & 97 & $84.7-108.3$ & 116.3 & 11.2 \\
\hline BENZO(g.h.i)PERYLENE* & 25.5 & & 132.2 & 8.7 \\
\hline INDENO(1.2.3-cd)PYRENE & 32 & $23.9-40$ & 97.4 & 6.6 \\
\hline DIBENZO(a.h)ANTHRACENE* & 14.2 & & 85.2 & 8.6 \\
\hline
\end{tabular}

* Compounds with non certified value.

Table V. PSE percent recoveries of PAH from urban dust SRM 1649. The number of determinations was 4 .

\begin{tabular}{lccc}
\hline & $\begin{array}{c}\text { Reference } \\
\text { Value }(\mu \mathrm{g} / \mathrm{g})\end{array}$ & $\begin{array}{c}\text { Recovery } \\
(\%)\end{array}$ & $\begin{array}{c}\text { RSD } \\
(\%)\end{array}$ \\
\hline PHENANTHRENE* & {$[4.5]$} & 94 & 6.8 \\
FLUORANTHENE & $7.1 \pm 0.5$ & 90 & 6.4 \\
PYRENE* & {$[6.6]$} & 84 & 9.8 \\
BENZO(a)ANTHRACENE & $2.6 \pm 0.3$ & 88 & 9.3 \\
CHRYSENE* & {$[3.6]$} & 126 & 7.1 \\
BENZO(b,k)FLUORANTHENE* & {$[8.2]$} & 109 & 5.2 \\
BENZO(a)PYRENE & $2.9 \pm 0.5$ & 80 & 5.8 \\
BENZO(g,h,i)PERYLENE & $4.5 \pm 1.1$ & 76 & 1.7 \\
INDENO(1,2,3-cd)PYRENE & $3.3 \pm 0.5$ & 76 & 4.6 \\
\hline
\end{tabular}

* Compounds with non certified value.

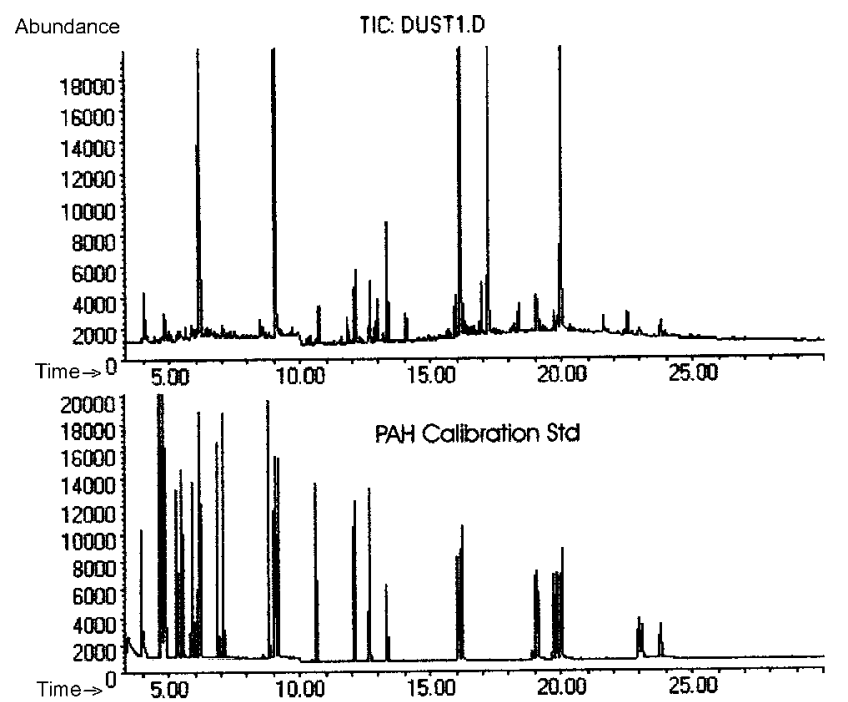

Fig. 4. Total ion chromatogram of: a) Urban Dust (SRM 1649) by PSE, b) Direct injection of PAH calibration standard. results obtained using MAE. These techniques have shown to be more powerful than the classical ultrasonic extraction. Savings in time, solvent usage and labour are possible due to the high extraction efficiencies of working at high temperature/pressure conditions. An added advantage of PSE is method development time is shortened significantly because of the simplicity of the process.

\section{Acknowledgements}

One of the authors (MPL) is indebted to Xunta de Galicia for a post-doctoral grant and to Environment Canada for its hospitality during the course of this work.

\section{References}

1. Hawthorne, S. B. Anal. Chem. 1990, 62, 633A.

2. Wenclawiak, B. Analysis with Supercritical Fluids: Extraction an Chromatography, Springer-Verlag, Berlin, Germany, 1992.

3. Luque de Castro, M. D.; Valcarcel, M.; Tena, M.T. Extraccion con fluidos supercriticos en el proceso analitico, ed. Reverte, Barcelona, Spain, 1993.

4. Chester, T. L.; Pinkston, J. D.; Raynie, D. E. Anal. Chem. 1992, 64, 153R.

5. Langenfeld, J. J.; Hawthorne, S. B.; Miller, D. J.; Pawliszyn, J. Anal. Chem. 1994, 66, 909.

6. Llompart, M.; Lorenzo, R. A.; Cela, R.; Li, K.; Belanger, J. M. R.; Pare, J. R. J. J. Chromatogr. 1997, 774, 243.

7. Ganzler, K.; Salgo, A.; Valko, K. J. Chromatogr. 1986, 371, 299.

8. Lopez-Avila, V.; Young, R.; Beckert, W. Anal. Chem. 1994, 66, 1097.

9. Lopez-Avila, V.; Young, R.; Teplitsky, N. J. AOAC Int. 1996, 79, 142.

10. Richter, B. E.; Ezzell, J. L.; Felix, D.; Roberts, K. A.; Later, D. W. Am. Lab., 1995, 27, 24.

11. Ezzell, J. L.; Richter, B. E.; Felix, D.; Black, S. R.; Meikle, J. E. $L C-G C$ 1995, 13, 390.

12. Richter, B. E.; Jones, B. A.; Ezzell, J. L.; Porter, N. L.; Avdalovic, N.; Pohl, C. Anal. Chem. 1996, 68, 1033.

13. Fisher, J. A.; Scarlett, M. J.; Stott, A. D. Environ. Sci. Technol. 1997, 31, 1120.

14. Kreisselmeier, A.; Durbeck, H. W. J. Chromatogr. 1997, 775, 187. 